\title{
In-Situ Synthesis of Temperature-Sensitive Hollow Microspheres via Interfacial Polymerization
}

\author{
Qunhui Sun and Yulin Deng* \\ School of Chemical \& Biomolecular Engineering and IPST at GT, \\ Georgia Institute of Technology, Atlanta, GA 30332-0620 \\ Email: Yulin.Deng@chbe.gatech.edu

\section{SUPPORTING INFORMATION}

\section{Materials and instrumentation:}

Materials: Sorbitan monooleate (Span-80), divinylbenzene (DVB, technical grade, 80\%, mixture of isomers), benzoyl peroxide (BPO, 97\%), tetraethylenepentamine (TEPA, technical grade), and toluene (99.8\%) were purchased from Sigma-Aldrich, and used without further purification. The $N$-isopropylacrylamide (NIPAM, 97\%) was purchased from Sigma-Aldrich and recrystallized from benzene and hexane twice and used after vacuum drying. ${ }^{1}$ De-ionized (DI) water (milli-Q grade) was used throughout the experiments.

Preparation of Hollow Microspheres: In a typical run, $4.0 \mathrm{~g}$ of Span-80, $0.2 \mathrm{~g}$ of DVB, and $0.2 \mathrm{~g}$ of BPO were dissolved in $50 \mathrm{ml}$ of toluene as the oil phase, and $1.0 \mathrm{~g}$ of NIPAM and $0.1 \mathrm{~g}$ of TEPA were dissolved in $5.0 \mathrm{ml}$ of DI water as the water phase. The two individual phases were mechanically mixed in a blender (Hamilton Beach brand, model 52200, from Proctor-Silex, Inc.) at Liquefy grade for $5 \mathrm{~min}$. The mixture was then poured into a $250 \mathrm{ml}$ three-neck round-bottom flask equipped with a mechanical stirrer and a condenser. Under mechanical agitation $(500 \pm 10 \mathrm{rpm})$, the reaction was carried out at $60{ }^{\circ} \mathrm{C}$ for more than $4 \mathrm{hrs}$ under nitrogen atmosphere. After that, the oil phase was separated under centrifugation (Beckman 20) at 8,000 rpm for $10 \mathrm{~min}$, and the water phase was collected for further characterization. 
Cryo-Breaking of Hollow Microspheres: A cryo-breaking technique to tailored breaking the microspheres under ultrasonification in a liquid nitrogen medium was developed, and detailed as follows. A mother liquor containing the microspheres in a glass vial was solidified first by immersing into a liquid nitrogen medium and then partially melted by naturally exposing to the air. At this point, the vial was placed into an ultrasonification chamber, and let the tip of the ultrasonicator touching at the solid/liquid interface. The process was lasted for ca. $60 \mathrm{~s}$. After that, the sample was ready for subsequent characterization.

Instrumentation: Scanning Electron Microscopy (SEM) was conducted on a LEO 1530 thermally-assisted field emission (TFE) scanning electron microscope SEM machine with an acceleration voltage of $3 \mathrm{kV}$. The samples were lightly sputter coated a thin layer of $\mathrm{Au} / \mathrm{Pd}$ prior to the observation. Optical microscope observation was carried out on a Leica DMLM microscope equipped with a Leica DC 100 camera shot atop. Images taken with the camera shot was handled in a computer connected with a Leica IM 50 Image Manager. The samples were heated on the stage spontaneously with the optical observation and pictures taking. The ultrasonification was performed on a W-385 sonicator (Heat System-Ultrasonics, Inc., USA) with $1 \mathrm{~s}$ of cycle time, $70 \%$ of duty cycle, and an output of the microtip at level 5.

\section{Supplementary references:}

(1) Hu, T.; You, Y.; Pan, C.; Wu, C.; J. Phys. Chem. B. 2002, 106, 6659-6662. 


\section{Supplementary figure S1:}



A

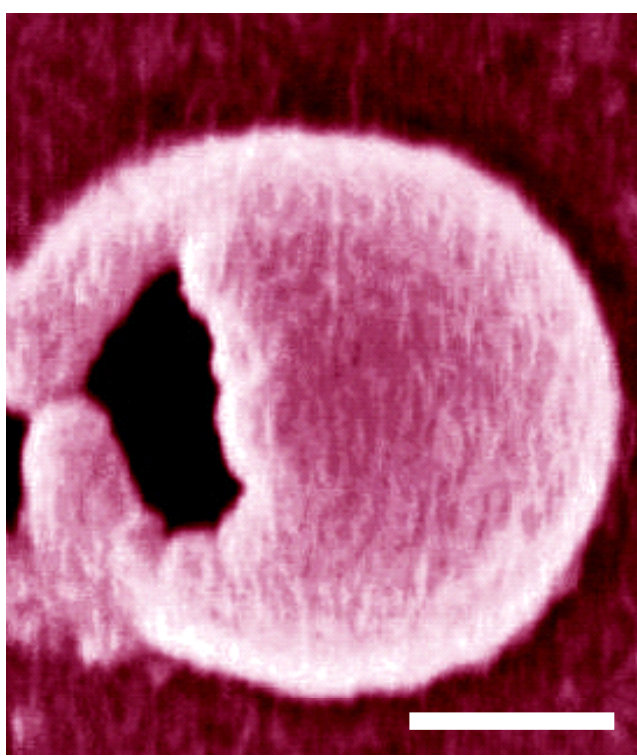

B

Figure S1. Supplementary overview SEM pictures of hollow microspheres before (A) and after (B) cryo-breaking. Scale bar: A: $2.0 \mu \mathrm{m} ; \mathrm{B}: 1.0 \mu \mathrm{m}$; 